\title{
Fuzzy Logic Based PID Auto Tuning Method of QNET 2.0 VTOL
}

\author{
Murk Junejo \\ Department of Electronic System Engineering, Mehran University of Engineering and Technology, \\ Jamshoro, Sindh, Pakistan \\ E-mail: murkfaizjunejo@gmail.com \\ Professor Dr. Arbab Nighat Kalhoro and Arsha Kumari \\ Department of Electronic System Engineering, Mehran University of Engineering and Technology, \\ Jamshoro, Sindh Pakistan. \\ E-mail: arbab.nighat@faculty.muet.edu.pk, arsharathi56@gmail.com
}

Received: 02 November 2019; Accepted: 23 November 2019; Published: 08 February 2020

\begin{abstract}
Unmanned aerial vehicles (UAVs) have gained a lot of attention from researchers due to their hovering and vertical take-off and landing. Different techniques and methods are being employed to implement UAVs. The QNET 2.0 VTOL board, specially designed for NI ELVIS II, is an important platform in the field of unmanned aerial vehicles (UAV). It is a helpful tool to demonstrate the essentials of vertical take-off and landing flight control (VTOL) at educational institutes. The PID controller installed in QNET 2.0 VTOL board is manually tuned is usually done by a skilled operator. This process of tuning is time-consuming and requires an expert's knowledge. Although PID control of various systems has been reported in the literature, its use is limited in nonlinear systems. For nonlinear systems. Fuzzy logic is suitable due to its nonlinearity capability. The purpose of this research is to study the dynamics of the QNET 2.0 VTOL model, simulate the flight control model in LabVIEW and to design an auto-tuned PID controller using Fuzzy logic for QNET 2.0 VTOL model in LabVIEW environment. This study shows that Fuzzy based autotuned PID controller controls the pitch angle of the QNET 2.0 VTOL model and gives promising results as compared to the existing PID controller in terms of autotuning in real-time and stability of the system.
\end{abstract}

Index Terms-Vertical take-off and landing (VTOL), QNET 2.0 VTOL, Pitch angle, Fuzzy based PID controller, Rule base, fuzzy algorithm.

\section{INTRODUCTION}

A rapid increase can be seen in the study of unmanned aerial vehicles' modeling and control. The use of UAVs is growing rapidly across many application domains including search rescue and security, providing wireless coverage, real-time monitoring, remote sensing, and surveillance [1]. This leads to the necessity of teaching the development of UAVs at educational institutes.
The QNET 2.0 VTOL board Fig.1 (a) is a trainer (a prototype model of the drone) developed by Quanser which introduces vertical take-off and landing (VTOL) [2]. The QNET 2.0 VTOL board is specially designed for NI ELVIS II Fig.1 (b) and can be programmed in the LabVIEW environment.

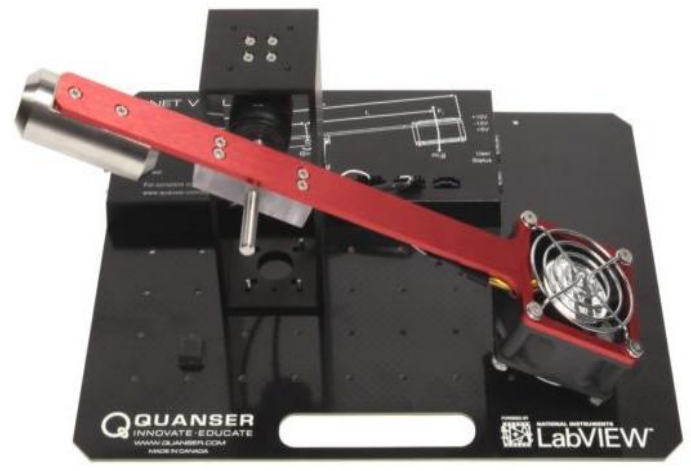

Fig.1 (a). QNET 2.0 VTOL board

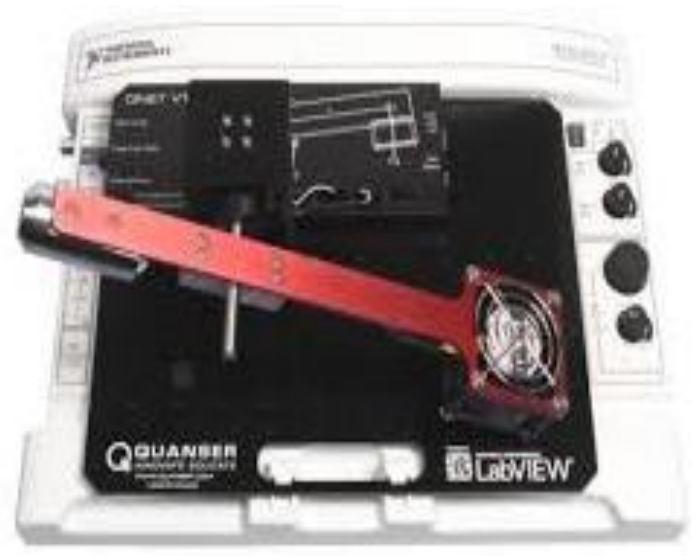

Fig.1 (b). QNET 2.0 VTOL board interfaced with NI ELVIS II

The built-in flight control model is simulated in LabVIEW and gains of the PID controller are adjusted manually to control the pitch angle of the QNET 2.0 VTOL 
board. The PID controller installed is manually tuned by adjusting the gains. This type of tuning requires an expert's knowledge to perfectly tune the PID controller and requires a lot of time. The major problem occurs if the parameters change abruptly, then the system gets unstable. The PID controller is mainly used for linear systems whereas the VTOL is a nonlinear system. To overcome these issues an auto-tuned Fuzzy based PID controller is presented.

With the help of a comparative study of different controllers used to implement UAVs, it is suggested that fuzzy logic control proves to provide better results in comparison to other controllers [3, 4, 5, 6, 7, 8, 9, 10, 11]. A new auto-tuning method using Fuzzy logic is introduced for tuning of the PID controller on the QNET 2.0 VOL board in LabVIEW. An intelligent algorithm using Fuzzy logic is built, which is capable of auto-tuning the PID controller by using its gain parameters. This tuning method not only will control the pitch angle of the system efficiently but will also reduce the effort of manual tuning as it offers auto-tuning of the controller gain.

The proposed research's main purpose is to control the pitch angle of QNET 2.0 VTOL. This research addresses the stabilization of the pitch angle of QNET 2.0 VTOL using a fuzzy-based tuning PID method. The main focuses of research include the study of QNET 2.0 VTOL model, design and implementation of fuzzy-based autotuned control logic to adjust intelligently the gain values i.e. $K_{p}, K_{i}$ and $K_{d}$ for a stable pitch angle and finally, validate the results using fuzzy control and existing PID control.

The paper is arranged in the following sequence: the literature review is presented in section II. In section III methodology, the system is studied thoroughly and gain parameters, at which the system gets stable pitch angle are found. The system's transfer function proves to help find out the gain parameters. Once the gain parameters are found then a fuzzy logic block is introduced in the system which takes in error and it's derivative as inputs and the gain parameters as outputs, which then are given to the PID controller. Based on these gain parameter values set of fuzzy rules are developed. Seven membership functions are chosen for all the inputs and outputs and their width is decided based on error and trial experience. Fuzzy blocks are built using these fuzzy rules. The fuzzy blocks replace the position control parameters block and introduce the auto-tuning of the controller gains. In section IV and V, the results are validated with the help of the system identification toolbox in MATLAB, which regenerates the response of the system.

\section{LITERATURE REVIEW}

In paper [4] work on the tuning of Quadrotor for attitude control utilizing the double-loop PID controller is proposed. The fictitious reference iterative tuning (FRIT) is used to tune the quadrotor. This tuning method for the quadrotor provides a systematic and fast way for finding the optimal gains of the quadrotor. Using one-shot experimental input-output data of every loop, the optimal gains help to get the attitude response of the quadrotor to replicate a reference response as much as possible. The proposed algorithm was validated with the help of experimentation and demonstrated significant performance improvement. Work was carried out in MATLAB environment on fuzzy logic control for UAV quadcopter.

In paper [5] authors presented an intelligent fuzzybased flight control for an autonomous quadrotor UAV. The modeling and control of Quadrotor UAV were taken into account in this research. With the help of MATLAB's fuzzy logic toolbox, an intelligent fuzzy flight controller was built. They compared the response of Fuzzy control against classical PID control in a MATLAB based environment, where fuzzy control showed better performance than classical PID.

In the study [6] authors designed an intelligent fuzzy system for an F-14 aero plane's longitudinal control. A fuzzy controller was designed to maintain the aircraft's stable horizontal flight by controlling only the elevation movements. The proposed controller was tested under noisy conditions and compared the results of the Fuzzy system against the PID controller in a simulation-based environment. A better result was obtained with the use of genetic algorithm optimization with a triangular membership function.

The paper [9] is presented for controlling the altitude dynamics of the Aerosonde UAV, an adaptive PID flight controller based on the parameter optimization fuzzy inference was designed. The online fuzzy inference is utilized as a self-adaptive mechanism to tune the PID parameters. The results of the adaptive PID flight controller showed improvement as compared to the fuzzy-based controller and genetically tuned PID controller.

In work-study [10] authors presented an autonomous path tracking and disturbance force rejection of UAV by introducing a fuzzy-based auto-tuned PID controller. A conventional PID controller and a fuzzy logic-based autotuning PID controller were compared and applied on the simulation-based model of a quadrotor mechanism with no sinusoidal force, disturbance force and payload variation in the system.

In the study [11] authors proposed research work on PID controllers' performance optimization by utilizing fuzzy logic. The authors presented a tuning method for gain parameters of PID controller using fuzzy logic and carried out work on a simulation-based model. The PID Controller is first tuned with the Ziegler Nichols tuning method, then using Relay auto-tuning and finally using fuzzy logic. When fuzzy logic tuning is used, the output response drastically improves.

From the literature review, it can be observed that most of the work has been done in a simulation environment using a conventional PID and Fuzzy controller. The recent study shows that the fuzzy-based PID controller gives satisfactory results when collated with a conventional PID controller. Most of the literature review addressed the fuzzy-based PID tuning method for height and orientation control whereas this research proposes a fuzzy-based PID tuning method for pitch angle stabilization in a real-time environment. 


\section{METHODOLOGY}

The block diagram of the Proposed Fuzzy Logic-based PID Auto-tuning method of QNET 2.0 VTOL is shown in Fig. 2. The system is a closed-loop system. And it can be seen that a fuzzy logic block is introduced in the system. The fuzzy logic block in an intelligent algorithm, which takes in error and it's derivative as two inputs and provides the tuned gain values i.e. $\mathrm{Kp}, \mathrm{Ki}$ and $\mathrm{Kd}$ to the PID controller. The PID controller takes in the tuned gain values as inputs and acts accordingly. The new Fuzzy based PID control can be implemented in LabVIEW to improve the performance of the QNET 2.0 VTOL board.

Therefore, the research focuses on the approach of using Fuzzy logic to auto-tune the PID controller to get better results.

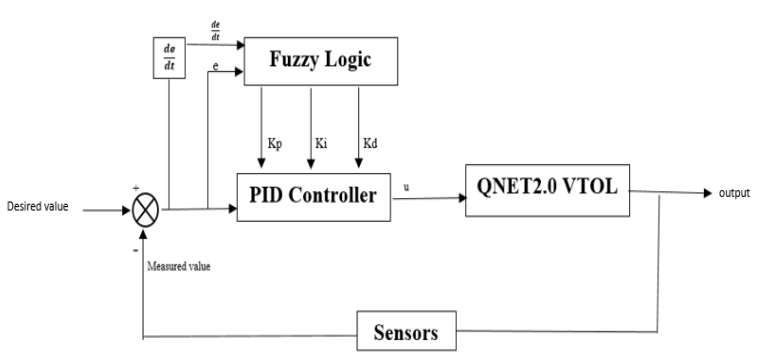

Fig.2. Proposed Fuzzy Logic Based PID Auto-tuning method of QNET 2.0 VTOL

With the help of a literature review $[2,13,14,15,16$, $17,18]$ the proposed research methodology is carried out by the following steps.

Step 1: Study existing PID control QNET 2.0 VTOL board in the LabVIEW environment and find out the gain values.

Step 2: Simulate the flight control model.

Step 3: Design an intelligent algorithm to auto-tune the PID gains using Fuzzy logic.

Step 4: To implement the proposed algorithm on QNET 2.0 VTOL board.

Step 5: PID controller V/S Fuzzy based auto-tuned PID Controller.

The research focuses on first finding the gain parameters where the system gets stable using its transfer function. The gain values and error are then used to develop a fuzzy rule base for $\mathrm{Kp}, \mathrm{Ki}$ and $\mathrm{Kd}$ using the Fuzzy system designer tool in the Lab VIEW environment. The fuzzy system is designed, implemented, and the simulations are also taken in the LabVIEW environment. The fuzzybased auto-tuned PID control's results are compared with the existing PID controller.

\section{A. Calculating The Gain Parameters}

The gain parameters i.e. Kp, Ki, and Kd where the system becomes stable are calculated with the help of the transfer function of QNET VTOL. The closed-loop transfer function of the system, from the position of reference $r$ to the angular VTOL position output $\theta$ is [2]:

$$
\frac{Y(S)}{R(S)}=\frac{\omega_{n}^{2}}{s^{2}+2 \zeta \omega_{n} s+\omega_{n}^{2}}
$$

The prototype third-order characteristic polynomial equation is:

$$
\begin{aligned}
& \left(s^{2}+2 \zeta \omega_{n} s+\omega_{n}^{2}\right)\left(s+p_{o}\right) \\
& =s^{3}+\left(2 \zeta \omega_{n}+p_{o}\right) s^{2}+\left(\omega_{n}^{2}+2 \zeta \omega_{n} p_{o}\right) s+\omega_{n}^{2} p_{o}
\end{aligned}
$$

With the help of eq. 1 and eq. 2, we can find values of the system gains i.e. $\mathrm{Kp}, \mathrm{Ki}$, and $\mathrm{Kd}$.

For Kp;

$$
\begin{gathered}
\left(k+k_{t} k_{p}\right) s=\left(\omega_{n}^{2}+2 p_{o} \zeta \omega_{n}\right) s \\
k+k_{t} k_{p}=\omega_{n}^{2}+2 p_{o} \zeta \omega_{n} \\
k_{p}=\frac{-k+2 p_{o} \zeta \omega_{n} J+\omega_{n}^{2} J}{k_{t}}
\end{gathered}
$$

For Ki;

$$
\begin{aligned}
& k_{t} k_{i}=p_{o} \omega_{n}^{2} \\
& k_{i}=\frac{p_{o} \omega_{n}^{2} J}{k_{t}}
\end{aligned}
$$

For Kd;

$$
\begin{aligned}
& \left(\beta+k_{t} k_{d}\right) s^{2}=\left(2 \zeta \omega_{n}+p_{o}\right) s^{2} \\
& \beta+k_{t} k_{d}=2 \zeta \omega_{n}+p_{o} \\
& k_{d}=\frac{-\beta+p_{o} J+2 \zeta \omega_{n} J}{k_{t}}
\end{aligned}
$$

Using the above equations $\mathrm{Kp}, \mathrm{Ki}$ and $\mathrm{Kd}$ are found i.e. $\mathrm{Kp}=0.0747, \mathrm{Ki}=0.3588$ and $\mathrm{Kd}=0.1409$ (set 1 ). With a common understanding, it can be concluded that to decrease overshoot increase $\mathrm{Kd}$, for better tracking increase $\mathrm{Ki}$ and for faster response increase $\mathrm{Kp}$.

Based on error and trial experience, the values for $\mathrm{Kp}$, $\mathrm{Ki}$, and $\mathrm{Kd}$ are taken as $\mathrm{Kp}=0.1, \mathrm{Ki}=0.4$ and $\mathrm{Kd}=0.2$ (set). The difference between the responses of two sets of values for $\mathrm{Kp}, \mathrm{Ki}$, and $\mathrm{Kd}$ are shown in Fig.3 (a) and Fig.3 (b).

The responses are taken in the LabVIEW environment, as it is used to interface the QNET 2.0 VTOL hardware. It can be seen that the obtained set1 of gain values can control the pitch angle of the system but set 2 of values is utilized the system response gets smoother and better as compared to the set 1 's response. Thus set 2 is used to further develop the fuzzy rule base. 


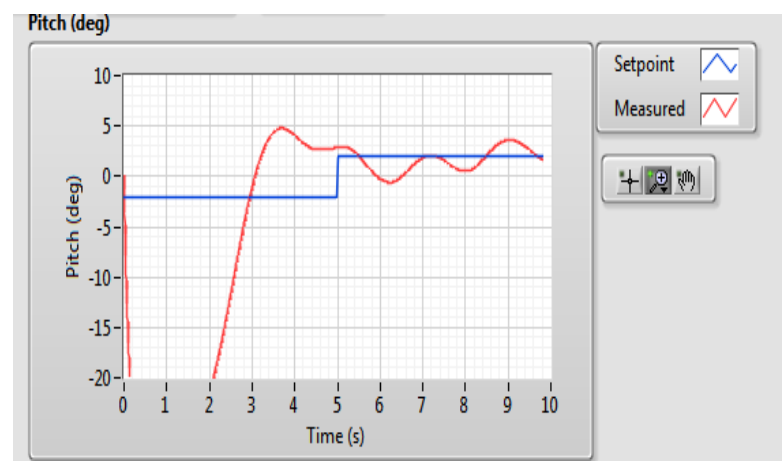

Fig. 3 (a). $\mathrm{Kp}=0.0747, \mathrm{Ki}=0.3588$ and $\mathrm{Kd}=0.1409$

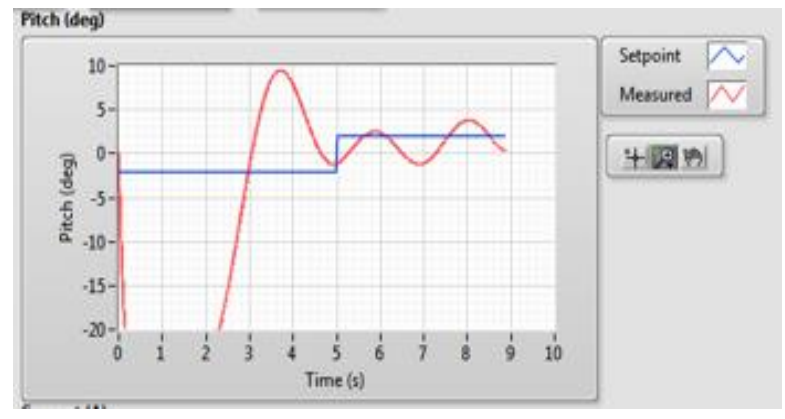

Fig. 3 (b). $\mathrm{Kp}=0.1, \mathrm{Ki}=0.4$ and $\mathrm{Kd}=0.2$

\section{B. Building The Fuzzy Logic Rule Base}

To build the fuzzy logic rule base, error (e) and error's derivative (ed) are taken as inputs of the fuzzy controller whereas $\mathrm{Kp}, \mathrm{Ki}$, and $\mathrm{Kd}$ are the fuzzy controller's outputs. Based on different values of inputs and outputs, seven membership functions for every input and output are chosen. The membership functions and their respective symbols can be seen in Table 1 .

Table 1. Membership functions and their symbols

\begin{tabular}{|c|c|c|c|}
\hline \multicolumn{2}{|c|}{ Input } & \multicolumn{2}{c|}{ Output } \\
\hline $\begin{array}{c}\text { Membership } \\
\text { function }\end{array}$ & Symbol & $\begin{array}{c}\text { Membership } \\
\text { function }\end{array}$ & Symbol \\
\hline Negative big & NB & Very very big & VVB \\
\hline Negative medium & NM & Very big & VB \\
\hline Negative small & NS & Big & B \\
\hline Zero & Z & Medium & M \\
\hline Positive small & PS & Small & S \\
\hline Positive medium & PM & Very small & VS \\
\hline Positive big & PB & Very very small & VVS \\
\hline
\end{tabular}

All the membership functions are chosen of triangular type and are identical, but the width of fuzzy sets is different for all the inputs and outputs.

The width of fuzzy sets is determined by error and trial experience and is chosen as:

- $\quad \mathrm{e}=\left[\begin{array}{ll}-0.4 & 0.4\end{array}\right]$

- $\mathrm{ed}=\left[\begin{array}{ll}-4 & 4\end{array}\right]$

- $\mathrm{Kp}=\left[\begin{array}{l}-0.00120 .2012] \\ 0.1940 .5236]\end{array}\right.$

- $\mathrm{Ki}=\left[\begin{array}{l}0.1940 .5236 \\ 0\end{array}\right.$

- $\mathrm{Kd}=\left[\begin{array}{ll}-0.0955 & 0.3773\end{array}\right]$
A set of forty-nine rules is developed for individual gain value by using seven membership functions of error and seven membership functions of error's derivative. These rules are designed based on the width of fuzzy sets and their behavior with the system.

Fuzzy rules for $\mathrm{Kp}, \mathrm{Ki}$ are shown in Table 2, and for Kd are shown in Table 3.

Table 2. Rule base for $\mathrm{Kp}$ and $\mathrm{Ki}$

\begin{tabular}{|c|c|c|c|c|c|c|c|}
\hline \multicolumn{7}{|c|}{ Error } \\
\hline & NB & NM & NS & ZO & PS & PM & PB \\
\hline NB & M & S & VS & VVS & VS & S & M \\
\hline NM & B & M & S & VS & S & M & B \\
\hline NS & VB & B & M & S & M & B & VB \\
\hline ZO & VVB & VB & B & M & B & VB & VVB \\
\hline PS & VB & B & M & S & M & B & VB \\
\hline PM & B & M & S & VS & S & M & B \\
\hline PB & M & S & VS & VVS & VS & S & M \\
\hline
\end{tabular}

Table 3. Rule base for Kd.

\begin{tabular}{|c|c|c|c|c|c|c|c|}
\hline \multicolumn{7}{|c|}{ Error } \\
\hline & NB & NM & NS & ZO & PS & PM & PB \\
\hline NB & M & B & VB & VVB & VB & B & M \\
\hline NM & S & M & B & VB & B & M & S \\
\hline NS & VS & S & M & B & M & S & VS \\
\hline ZO & VVS & VS & S & M & S & VS & VVS \\
\hline PS & VS & S & M & B & M & S & VS \\
\hline PM & S & M & B & VB & B & M & S \\
\hline PB & M & B & VB & VVB & VB & B & M \\
\hline
\end{tabular}

The rule bases are built with the help of the Fuzzy system designer tool in LabVIEW. This tool helps to design fuzzy systems. There are three sections of the Fuzzy system designer tool i.e. Variables, Rules, and Test system. After defining the input and output variables and the width of the membership functions in the "variable" section of the fuzzy system designer Fig. 4, the rules are then defined in the "Rules" section. The rules define the fuzzy systems' behavior. The system can also be tested based on given input values.

After the rule base for $\mathrm{Kp}, \mathrm{Ki}$ and $\mathrm{Kd}$ are built with Fuzzy system designers. The fuzzy system is saved in the .fs file format. "The FL Load Fuzzy system VI" Fig. 5 and "FL Fuzzy controller VI" Fig. 6, are used to load the .fs file and use it further.

The .fs file is loaded with the FL Load Fuzzy system VI. The .fs file can be fetched by providing the path of the file as input to the FL Load Fuzzy system VI. The "fuzzy system out" output of the FL Load Fuzzy system VI is connected to the "fuzzy system in" input of the FL Fuzzy controller (MISO) VI, which then provides the input to PID controller. These VI connections can be seen in Fig. 7. 


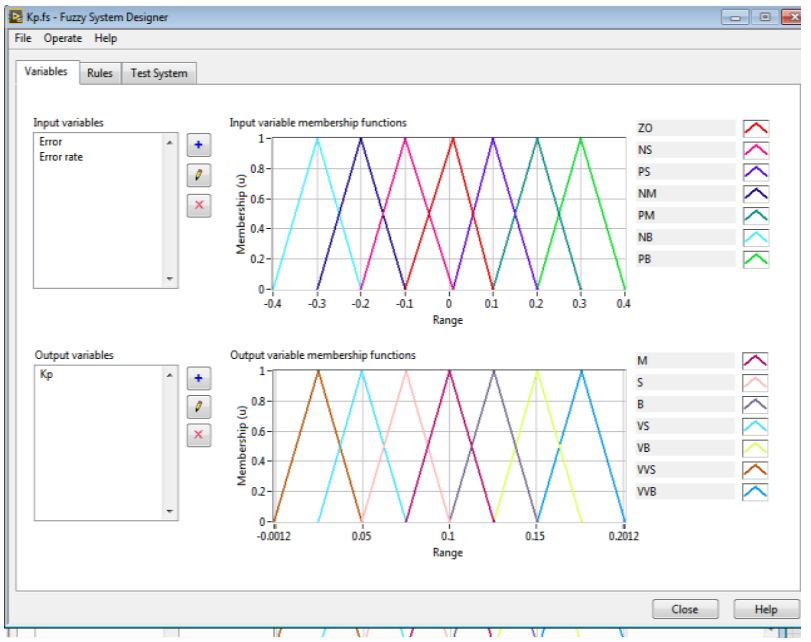

Fig.4. Fuzzy System Designer

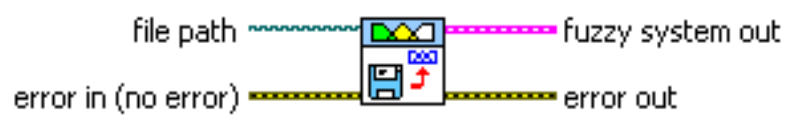

Fig.5. FL Load Fuzzy system VI

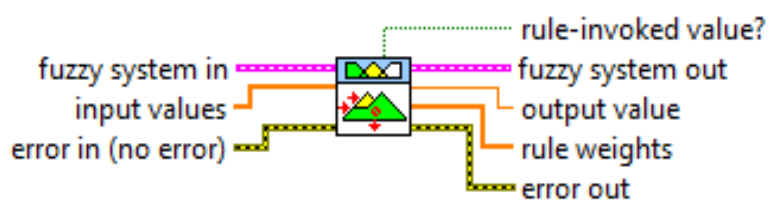

Fig.6. FL Fuzzy controller VI

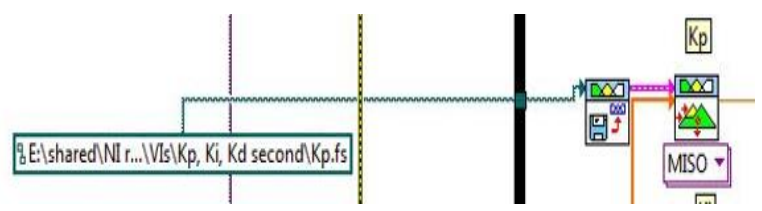

Fig.7. VI connections

The front panel diagram and block diagram of existing QNET 2.0 VTOL Flight control are shown in Fig.8 (a) and Fig.8 (b) respectively.

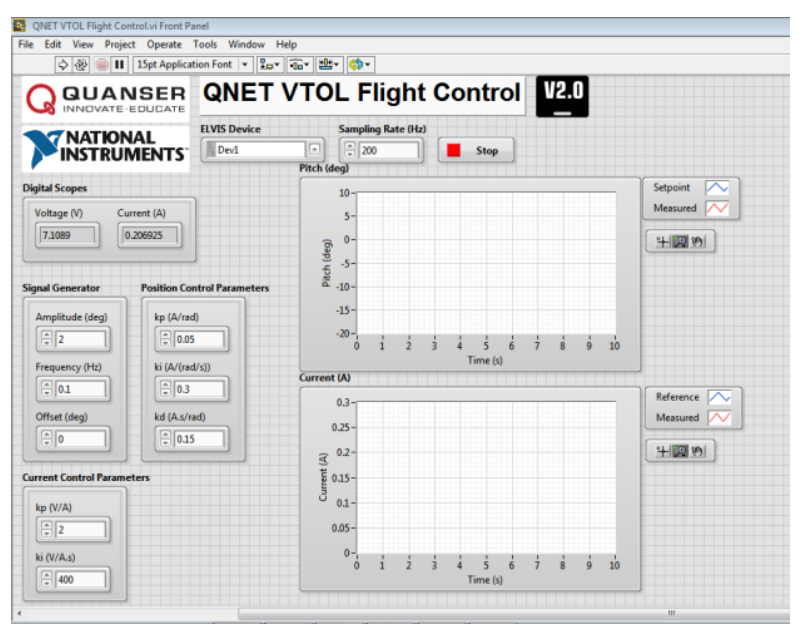

Fig.8 (a). QNET 2.0 VTOL Flight control front panel

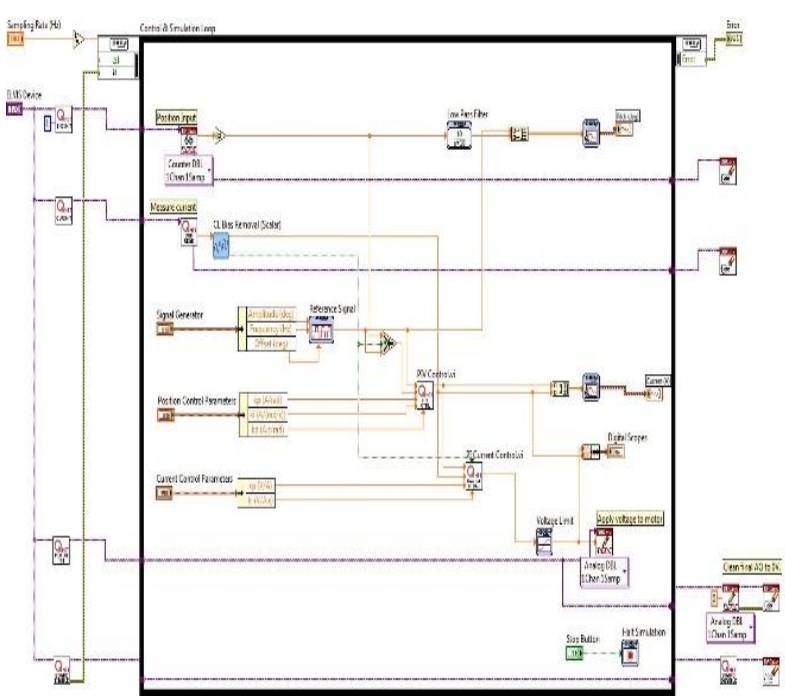

Fig.8 (b). QNET 2.0 VTOL Flight control block diagram

And the front panel and block diagram of implemented fuzzy-based auto-tuned PID controller is shown in Fig.9 (a) and Fig.9 (b) respectively.

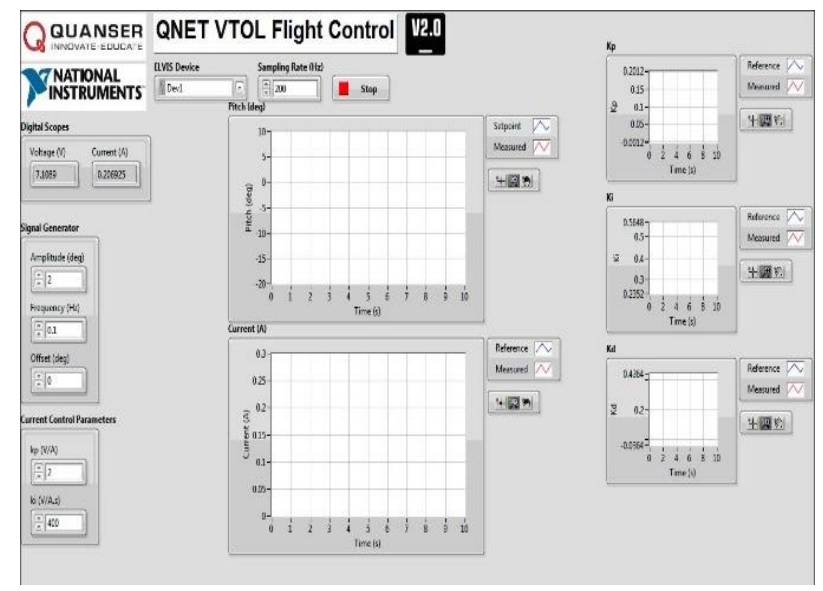

Fig.9 (a). Fuzzy controller front panel

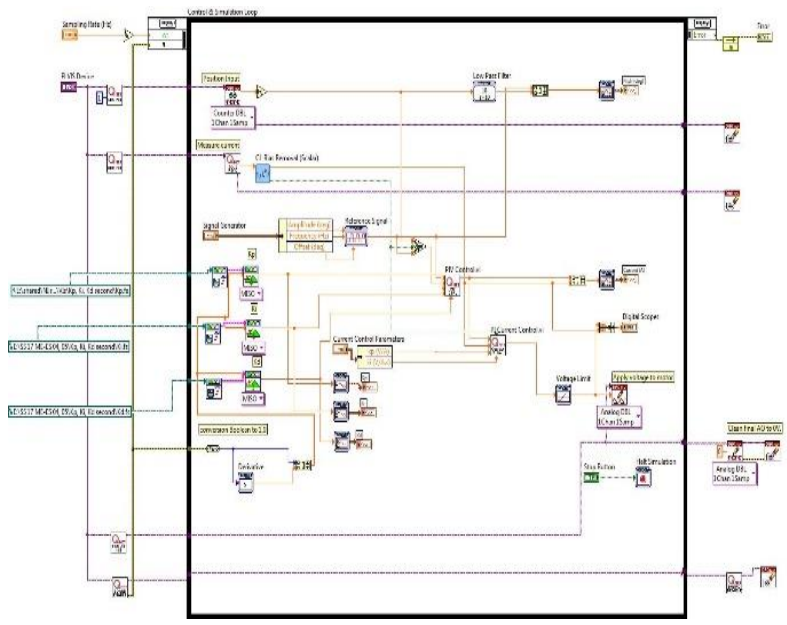

Fig.9 (b). Fuzzy controller block diagram 
In the block diagram of existing QNET VTOL Flight control the block "position control parameters" which is the input block for $\mathrm{kp}, \mathrm{ki}$, and $\mathrm{kd}$, is replaced by fuzzy logic blocks in the block diagram of the fuzzy-based auto-tuned PID controller to intelligently control the parameters. And the output of the fuzzy blocks is given as input to the PID controller.

\section{SiMULATION AND RESUlTS}

To validate the results four characteristics of the step response are taken into consideration i.e. Rise time, settling time, overshoot and Peak time. The result of existing QNET VTOL Flight control can be seen in Fig.10 (a) and the step characteristics can be found as, rise time $=1.7 \mathrm{~s}$, peak time $=3.9 \mathrm{~s}$, settling time $=9.4 \mathrm{~s}$ and overshoot $=$ $40 \%$.

Whereas the result of a fuzzy logic-based auto-tuned PID controller can be seen in Fig.10 (b) and the step characteristics can be found as, rise time $=1.46 \mathrm{~s}$, peak time $=3.6 \mathrm{~s}$, settling time $=8.54 \mathrm{~s}$ and overshoot $=10 \%$.

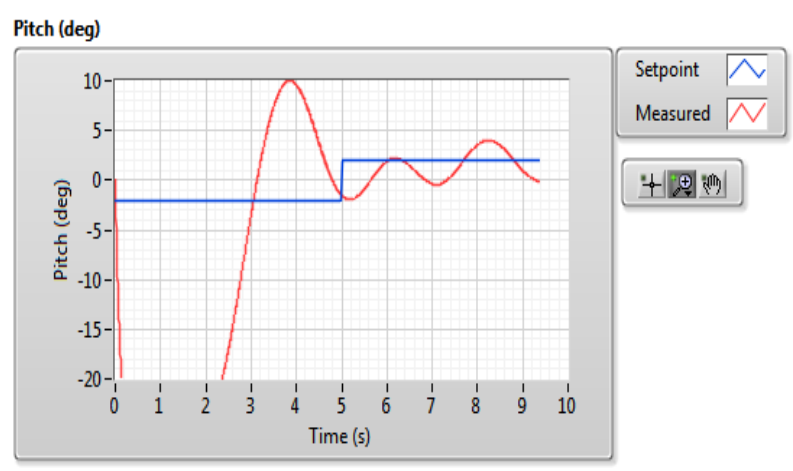

Fig.10 (a). PID Controller result

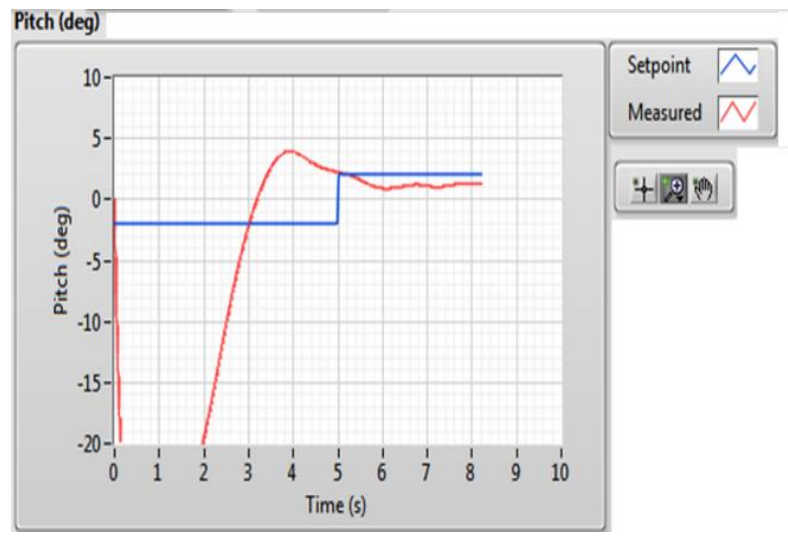

Fig.10 (b). Fuzzy controller result

System Identification toolbox Fig.11 is used in MATLAB to regenerate the results of both the existing PID control and the fuzzy logic-based auto-tuned PID control to compare and validate the results. This toolbox helps to regenerate the results with the help of the system's transfer function.

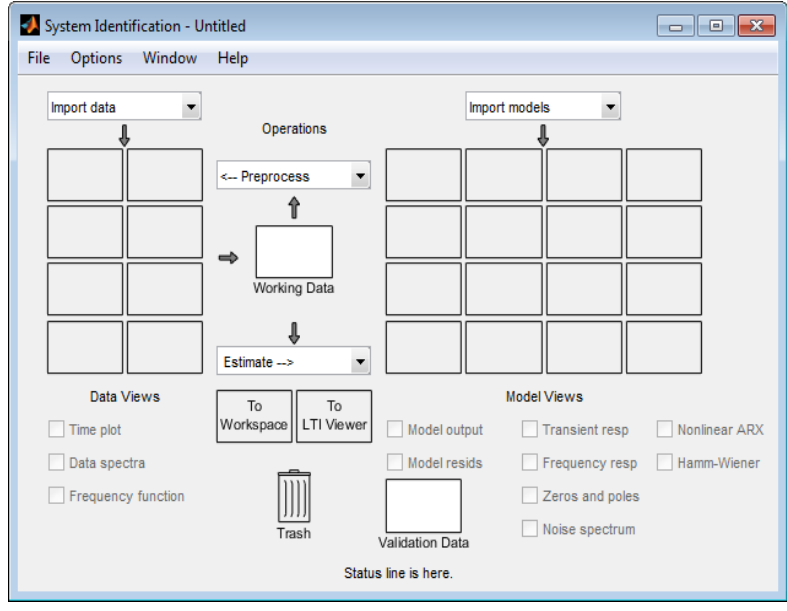

Fig.11. System Identification toolbox

System Identification toolbox regenerated the results for the system with an $80 \%$ approximation and this comparison can be seen in Fig.12. The response of the fuzzybased PID controller is better than the existing PID controller.

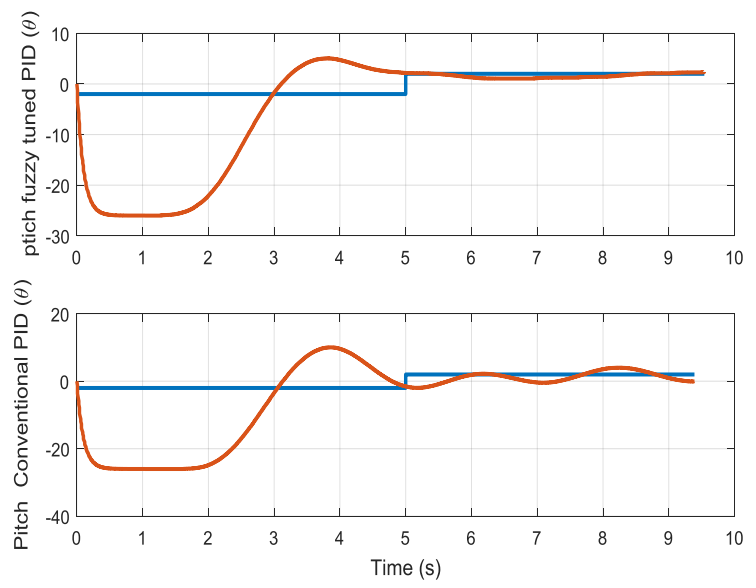

Fig.12. MATLAB simulation

While in Fig.13 (a) and Fig.13 (b) the step characteristics can be seen on the step response of the fuzzy logicbased auto-tuned PID controller.

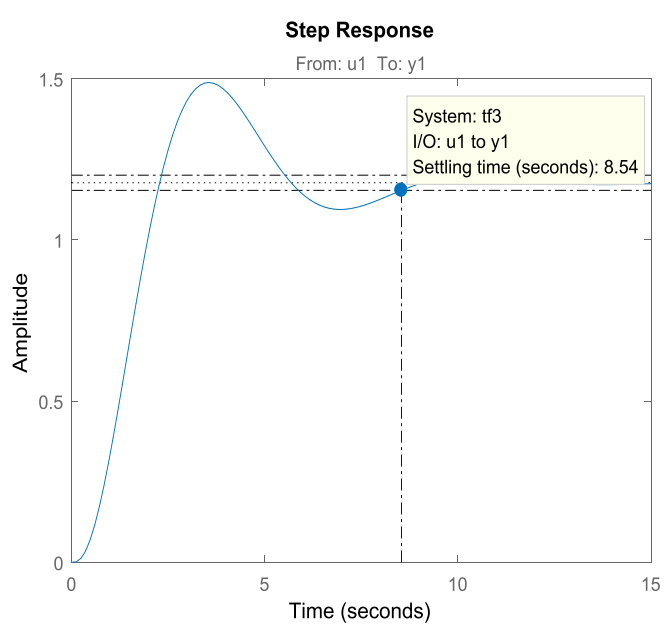

Fig.13 (a). Step Response 


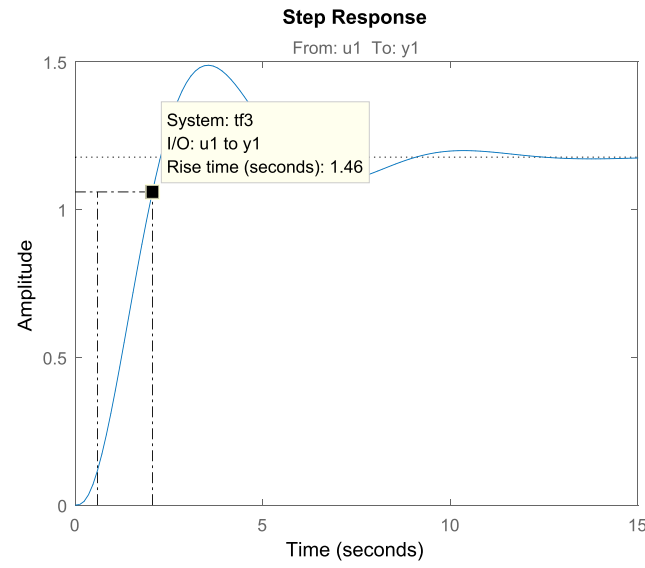

Fig.13 (b). Step Response

Table 4. Step characteristics

\begin{tabular}{|c|c|c|}
\hline Characteristics & PID Controller & Fuzzy PID Controller \\
\hline Rise time & $1.7 \mathrm{~S}$ & $1,46 \mathrm{~S}$ \\
\hline Peak time & $3.9 \mathrm{~s}$ & $3.6 \mathrm{~S}$ \\
\hline Settling time & $9.4 \mathrm{~s}$ & $8.54 \mathrm{~s}$ \\
\hline Overshoot & $40 \%$ & $10 \%$ \\
\hline
\end{tabular}

A decrease in all the characteristics of the step response is observed. It is clear from Table 4 that the fuzzy logic based auto-tuned PID controller gives promising results as compared to the existing PID controller. It cannot be denied that the result has drastically improved, and the response of the system is smoother and better after the implementation of the fuzzy-based PID auto-tuning method.

\section{CONCLUSION AND FUTURE WORK}

The fuzzy-based auto-tuning method is introduced to tune the installed PID controller of QNET 2.0 VTOL. The fuzzy-based auto-tuned controller is compared with the manually tuned PID controller installed on the system. The comparison is based on the simulation results of the QNET 2.0 VTOL model taken in the LabVIEW environment. The comparison is done based on step characteristics i.e. Peak time, Rise time, Settling time and Overshoot. The response shows that the Fuzzy based auto-tuned PID controller can control the pitch angle of the QNET 2.0 VTOL. From the results, it can be observed that on given values of controller gains; significant performance is improved with the Fuzzy based PID controller than the existing PID controller. It can also be observed that the values for peak time, rise time, percent overshoot and settling time have reduced with Fuzzy based auto-tuned PID controller.

The existing PID controller is manually tuned. Whereas, Fuzzy based PID controller tuning method is autotuned and requires less expertise and is stable. The proposed fuzzy auto-tuning PID method gives promising results as compared to the existing PID controller for a stable pitch angle of QNET 2.0 VTOL.

In future, the proposed controller can be implemented and tested against different disturbances. The proposed control can also be implemented on a real UAV and perform different flight modes.

\section{ACKNOWLEDGEMENT}

A significant appreciate addressed to the Institute of Information and communication technologies, Mehran University of engineering and technology Jamshoro for providing the platform of research for students and to and Department of Electronic Engineering, Mehran University of engineering and technology Jamshoro for providing the QNET 2.0 VTOL board for research. This would have not been possible without guidance and support of family, friends, and teachers.

\section{REFERENCES}

[1] Hazim Shakhatreh, "Unmanned Aerial Vehicles: A Surve y on Civil Applications and Key Research Challenges," ar Xiv:1810.09729[cs.RO].

[2] QNET 2.0 VTOL Board Quanser", Quanser, 2011. Availa ble: https://www.quanser.com/products/qnet-2-0-vtol-boar d/.

[3] Zhangli Yang, Lei Li, and Buqing Liu, "Auto-tuning Meth od of Fuzzy PID Controller Parameter Based on Self-learn ing System," 11th International Conference on Fuzzy Syst ems and Knowledge Discovery, 2014.

[4] A. Julkananusart and I. Nilkhamhang, "Quadrotor tuning f or attitude control based on double-loop PID controller usi ng fictitious reference iterative tuning (FRIT)," in Proceed ings of the 41st Annual Conference of the IEEE Industrial Electronics Society, IECON 2015, pp. 4865-4870, Novem ber 2015.

[5] Vijaykumar Sureshkumar Fnu, Kelly Cohen, "Intelligent Fuzzy Flight Control of an Autonomous Quadrotor UAV", Conference paper - January 2014, DOI: 10.2514/6.2014-0 992.

[6] Leticia Cervantes and Oscar Castillo," Design of a Fuzzy System for the Longitudinal Control of an F-14 Airplane," soft computing for Intell. Control and Mob. The robot, 20 10.

[7] Arbab Nighat Khizer, Syed Amjad Ali and Dai-Yaping, " Stable Hovering Flight for a small unmanned Helicopter u sing Fuzzy control, "Hindawi Publishing Corporation Mat hematical Problems in Engineering, Vol 2014.

[8] Arbab Nighat Khizer Syed Amjad Ali and Dai-Yaping," S impler Fuzzy Logic Controller (SFLC) Design for 3DOF Laboratory scaled Helicopter," IJRRAS, Vol15Issue2, Ma y 2013.

[9] Amr Sarhan and Shiyin Qin," Adaptive PID Control of U AV Altitude Dynamics Based on Parameter Optimization with Fuzzy Inference,” International Journal of Modeling and Optimization, Vol. 6, No. 4, August 2016.

[10] Theerasak Sangyam, Pined Laohapiengsak, Wonlop Chon gcharoen, and Itthisek Nilkhamhang, "Autonomous path tr acking and disturbance force rejection of UAV using fuz zy-based auto-tuning PID controller," ECTI-CON2010: T he 2010 ECTI International Conference on Electrical Engi neering/ Electronics, Computer, Telecommunications and Information Technology, Chiang Mai, Thailand, 2010, pp. 528-531.

[11] S. M. Sam and T. S. Angel, "Performance optimization of PID controllers using fuzzy logic," 2017 IEEE Internation al Conference on Smart Technologies and Management $f$ or Computing, Communication, Controls, Energy and Mat erials (ICSTM), Chennai, 2017, pp. 438-442. 
[12] Lucio R. Riberio and Neusa Maria F. Oliveira, "UAV Aut opilot Controllers Test Platform Using Matlab/Simulink a nd X-Plane," $40^{\text {th }}$ ASEE/IEEE Frontiers in Education Con ference S2H-1, 2010.

[13] Tipsuwanporn et al, "Fuzzy Logic PID controller based on FPGA for process control," IEEE, 2004.

[14] Qingsi Zhang and Jinfeng Bai, “Application of Fuzzy Log ic controller with self-tuning PID parameters for the contr ol system of $40 \mathrm{Kg}$ coke oven," $3^{\text {rd }}$ International conferenc e on instrumentation, measurement, computer, communica tion and control, 2013.

[15] Seyed Jamal Haddadi and Payam Zarafshan, "Experiment al stability study of an Octrotor using an intelligent contro ller," IEEE 2016.

[16] Yu Jian and Liu Changliang, "Design of self-tuning PID c ontroller with Fuzzy variable parameters based on LabVI EW," International conference on information and automa tion Lijiang, China, 2015.

[17] Tri kuntoro Priyambodo and Andi Dharmawan, "Auto Ver tical takeoff and landing on quadrotor using PID-Fuzzy," Journal of Engineering and applied sciences 12(special iss ue 3), 2017.

[18] Ehsan. Abbasi, "Development and implementation of an a daptive fuzzy control system for a VTOL vehicle in hover ing mode," International Journal of control theory and co mputer modeling (IJCTCM) Vol 7, No.1/2, April 2017.

[19] Hooman Nasr Azadani and Roozbeh Torkzadeh, "Design of GA Optimized Fuzzy Logic-based PID Controller for $t$ he Two Area Non-Reheat Thermal Power System," $13^{\text {th }}$ Ir anian Conference on Fuzzy Systems (IFSC), 2013.

[20] Francesco Sabatino, "Quadrotor control: modeling, nonlin ear control design, and simulation", Master's Degree Proje ct Stockholm, Sweden June 2015.

\section{Authors' Profiles}

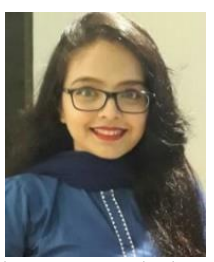

Murk Junejo - She has completed her B.E in 2016 from the Department of Electronic Engineering, Mehran University of Engineering and Technology, Jamshoro. She is currently pursuing her Master's in Electronic System Engineering from Mehran University of Engineering and Technology Jamshoro. She had been awarded a semester exchange scholarship (UGRAD Pakistan) in the United States of America in the year 2016, funded by USEFP. Her research interests include IoT systems, control systems, intelligent systems, and others.

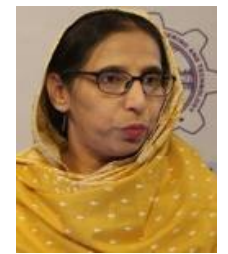

Professor Dr. Arbab Nighat Kalhoro - She is working with the Department of Electronic Engineering, Mehran UET, Jamshoro, Sindh, Pakistan. She obtained her B.E in 1997 and M.E in 2009 from the Department of Electronic Engineering Mehran University of Engineering Technology Jamshoro. She completed her Ph.D. In 2004 from Beijing Institute of Technology (BIT), China. She has 26 national and international publications.

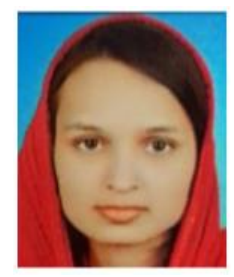

Arsha Kumari - She has completed her B.E in 2016 from the Department of Electronic Engineering Mehran University of Engineering Technology Jamshoro. Currently, she is doing her Master's in Electronic System Engineering from Mehran UET Jamshoro. She has done many internships related to her field from which she learnt a lot. Her research interests include Machine Learning, Computer Vision, and Deep Learning.

How to cite this paper: Murk Junejo, Arbab Nighat Kalhoro, Arsha Kumari, "Fuzzy Logic Based PID Auto Tuning Method of QNET 2.0 VTOL", International Journal of Information Technology and Computer Science(IJITCS), Vol.12, No.1, pp.9-16, 2020. DOI: 10.5815/ijitcs.2020.01.02 